\title{
Cross sections for the elastic scattering of low-energy electrons by molecular fluorine : an approximate theoretical treatment using discrete basis functions $\dagger$
}

\author{
T N Rescigno $\ddagger$, C F Bender $\ddagger$, C W McCurdy $\S \|$ and V McKoy $\S$ \\ \$ Lawrence Livermore Laboratory, University of California, Livermore, California 94550 , \\ USA \\ 8 Arthur Amos Noyes Laboratory of Chemical Physics, California Institute of Technology, \\ Pasadena, California 91125, USA
}

Received 12 January 1976, in final form 1 April 1976

\begin{abstract}
We present phaseshifts and total cross sections for the elastic scattering of low-energy $(0-13.6 \mathrm{eV})$ electrons by molecular fluorine. The phaseshifts are obtained by an approximate technique based on the weak asymptotic coupling of orbital angular momenta and are calculated solely from the results of a discrete basis set diagonalization of the molecular Hamiltonian. Correlation and polarization effects are not treated. The elastic cross section is dominated by a $\Sigma_{u}^{+}$shape resonance at about $2 \cdot 2 \mathrm{eV}$ in the staticexchange model.
\end{abstract}

\section{Introduction}

In a previous paper (McCurdy et al 1976, hereafter referred to as I) we outlined a method for obtaining approximate low-energy electron-diatomic-molecule elastic scattering cross sections solely from the results of a basis set Hartree-Fock calculation on the closed-shell molecular target. The method was based on the assumed validity of the so-called 'low $l$-spoiling' approximation (Fano 1970) of low-energy electronmolecule scattering, as well as the fact that the eigenfunctions at scattering energies obtained by diagonalizing the Hamiltonian in a sufficiently large set of squareintegrable basis functions are, apart from an overall normalization, equal to the true wavefunctions over the region spanned by the basis set (Hazi and Taylor 1970, Hazi and Fels 1971). These assumptions were tested by calculations on $\mathrm{e}^{-}-\mathrm{H}_{2}$ and $\mathrm{e}^{-}-\mathrm{N}_{2}$ and the results obtained were found to be in reasonably good agreement with more elaborate theoretical treatments. In this paper, the method of $I$ is applied to a study of low-energy $\mathrm{e}^{-}-\mathrm{F}_{2}$ elastic scattering.

Very little is known about the scattering of electrons by $F_{2}$, either experimentally or theoretically. To our knowledge, no previous theoretical studies of elastic $e^{-}-\mathrm{F}_{2}$ scattering have been performed. A rigorous treatment would be complicated by the

$\dagger$ This work was performed under the auspices of the US Energy Research and Development Administration under contract No W-7405-ENG-48.

II Present address: Department of Chemistry, University of California, Berkeley, California 94720, USA.

Supported by a grant from the National Science Foundation. 
fact that the ground state of $F_{2}$ is more difficult to describe accurately than the ground states of other first-row diatomics. The calculations presented here make use of the static-exchange approximation in which the $F_{2}$ target is frozen in its Hartree-Fock ground-state wavefunction. The well known failure of the HartreeFock wavefunction to predict $F_{2}$ to be bound relative to two fluorine atoms (Wahl 1964) raises some doubt as to the adequacy of a Hartree-Fock description for constructing a scattering potential. Thus, until more accurate calculations are performed, the present results should be considered preliminary.

Our calculations on this system incorporate a number of simplifying approximations. As in the computations reported in I, we have ignored correlation and polarization effects in restricting ourselves to a study of the static-exchange approximation with a Hartree-Fock target wavefunction. The dominant feature we find in the total elastic cross section is a $\Sigma_{\mathfrak{u}}^{+}$shape resonance coming from the p $\sigma$ phaseshift at about $2.2 \mathrm{eV}$. Before presenting our results, we give a brief summary of the procedure used in this work.

\section{Theory}

The method used to obtain electron-diatomic-molecule phaseshifts, as well as the nature and validity of the assumptions underlying the method, have been discussed fully in I and only a brief summary will be given here. Simply stated, the low $l$-spoiling assumption states that at low electron energies orbital angular momentum is approximately conserved in the body-fixed molecular frame. This conservation comes about from the repulsive centrifugal barrier of the effective potential which effectively prevents slow electrons from penetrating the highly non-spherical molecular core (Fano 1970). It was further argued that this approximation should hold particularly well for a homonuclear diatomic molecule, since in this case the centre of symmetry causes even values of $l$ to uncouple from odd values.

We stress that the low $l$-spoiling approximation does not imply that the continuum wavefunction is proportional to a particular spherical harmonic at small radial distances nor that a single-centre expansion of the molecular potential or the scattering wavefunction is rapidly convergent. It merely states that at low energies it should be possible to find continuum solutions asymptotically proportional to a particular partial wave.

To this end, we diagonalize the molecular Hamiltonian in a discrete set of Gaussian basis functions that give a non-vanishing contribution to the particular symmetry (labelled by $l$ and $|m|$ values) under consideration. This set is chosen to give a good description of the occupied Hartree-Fock molecular orbitals and is then augmented with additional diffuse functions of one particular symmetry, most of which are placed at the centre of the molecule. The molecular Hamiltonian is then diagonalized in this basis. For each positive energy eigenfunction, $\Psi_{E}(r)$, a radial wavefunction $R_{l m}^{E}(r)$ is obtained by projection with the appropriate spherical harmonic:

$$
R_{l m}^{E}(r)=\int \mathrm{d} \Omega Y_{l m}^{*}(\Omega) \Psi_{E}(\boldsymbol{r}) .
$$

For the particular case of a diatomic molecule with Gaussian basis functions chosen along the axis of symmetry the projection integral of equation (1) can be done analytically. 
On the assumption that the basis set representation of $R_{l m}^{E}(r)$ is valid over the region spanned by the basis (Hazi and Taylor 1970, Hazi and Fels 1971), and that this region is sufficiently large to reach a point where the potential has become vanishingly small, the tangent of the phaseshift may be obtained by plotting

$$
\tan \delta_{l m}(r)=-\frac{k R_{l m}^{E}(r) j_{l}^{\prime}(k r)-R_{l m}^{E^{\prime}}(r) j_{l}(k r)}{k R_{l m}^{E}(r) n_{l}^{\prime}(k r)-R_{l m}^{E^{\prime}}(r) n_{l}(k r)}
$$

as a function of $r$ and extracting a value for the phaseshift from the region where this function levels off to a constant value. This part of the procedure can be thought of as a basis set approach to the familiar variable-phase method of potential scattering (Calogero 1967). In contrast to the latter procedure, however, the value of $\tan \delta_{l m}(r)$ obtained from equation (2) will again vary as a function of $r$ when the basis is no longer capable of representing $R_{l m}^{E}(r)$ accurately.

The assumption of angular momentum decoupling simplifies the problem considerably since we do not have to face the prospect of dealing with more than one linearly independent regular solution at one energy for a particular choice of $l$ and $m$. Relaxation of this assumption would complicate the problem considerably (Hazi 1973).

\section{Results}

All of our calculations were carried out at the equilibrium internuclear separation of $\mathrm{F}_{2}, 2.68 a_{0}$. Correlation and polarization effects have not been considered in these computations; that is, the continuum wavefunctions were obtained as the positive energy eigenfunctions of the Hartree-Fock Hamiltonian of $F_{2}$. The HF occupied orbitals for $\mathrm{F}_{2}$ were computed using a basis of $[6 \mathrm{~s} / 5 \mathrm{p}]$ Gaussians on each fluorine contracted from a $(10 \mathrm{~s} / 6 \mathrm{p})$ set of primitives similar to that suggested by Dunning (1970), plus two $\mathrm{d} \sigma$ and two $\mathrm{d} \pi$ polarization functions (Dunning 1971) on each fluorine. The results of the self-consistent field ( $\mathrm{SCF}$ ) computation are summarized in table 1 .

Five separate diagonalizations were performed to obtain virtual Hartree-Fock eigenfunctions of $\Sigma_{\mathrm{g}}^{+}, \Sigma_{\mathrm{u}}^{+}, \Pi_{\mathrm{u}}, \Pi_{\mathrm{g}}$ and $\Delta_{\mathrm{g}}$ symmetry. For the $\Sigma_{\mathrm{g}}^{+}$orbitals, the previous basis was augmented by two s-type and two z-type Gaussians on each fluorine and eight more $\mathrm{s}$ functions at the midpoint. The $\Sigma_{\mathrm{u}}^{+}$calculations were done by adding

Table 1. SCF molecular orbital eigenvalues for $\mathrm{F}_{2} \dagger$. (Energies are given in au.)

\begin{tabular}{ll}
\hline$E_{\mathrm{HF}}$ & -198.73201 \\
$\epsilon_{1 \sigma_{\mathrm{g}}}$ & -26.4129 \\
$\epsilon_{2 \sigma_{\mathrm{s}}}$ & -1.7523 \\
$\epsilon_{3 \sigma_{\mathrm{g}}}$ & -0.7383 \\
$\epsilon_{1 \sigma_{\mathrm{u}}}$ & -26.4126 \\
$\epsilon_{2 \sigma_{\mathrm{u}}}$ & -1.4890 \\
$\epsilon_{1 \pi_{\mathrm{a}}}$ & -0.7984 \\
$\epsilon_{\mid \pi_{\mathrm{g}}}$ & -0.6563 \\
\hline
\end{tabular}

† In a $6 \mathrm{~s} / 5 \mathrm{p}$ basis of contracted Gaussian functions. This basis was only used to represent the occupied orbitals. 
Table 2. $\mathrm{e}^{-}-\mathrm{F}_{2}$ phaseshifts.

\begin{tabular}{|c|c|c|c|c|c|c|c|c|c|}
\hline \multicolumn{2}{|c|}{ s } & \multicolumn{2}{|c|}{$\mathrm{p} \sigma$} & \multicolumn{2}{|c|}{$\mathrm{p} \pi$} & \multicolumn{2}{|c|}{$\mathrm{d} \pi$} & \multicolumn{2}{|c|}{$\mathrm{d} \delta$} \\
\hline$k(\mathrm{au})$ & $\delta_{00}$ & $k(\mathrm{au})$ & $\delta_{10}$ & $k(\mathrm{au})$ & $\delta_{11}$ & $k(\mathrm{au})$ & $\delta_{21}$ & $k(\mathrm{au})$ & $\delta_{22}$ \\
\hline 0.0216 & $3 \cdot 1005$ & 0.0258 & 0.00001 & 0.0376 & $3 \cdot 14143$ & 0.0460 & $3 \cdot 1415917$ & 0.3880 & 0.07 \\
\hline 0.04619 & 3.054 & $0 \cdot 0480$ & 0.0001 & 0.0699 & $3 \cdot 14078$ & 0.0804 & $3 \cdot 141589$ & 0.5639 & 0.20 \\
\hline 0.07738 & $2 \cdot 994$ & 0.0736 & 0.002 & $0 \cdot 1110$ & $3 \cdot 1377$ & 0.1239 & $3 \cdot 141534$ & 0.8155 & 0.22 \\
\hline $0 \cdot 1199$ & $2 \cdot 914$ & 0.1147 & 0.004 & $0 \cdot 1671$ & $3 \cdot 1308$ & $0 \cdot 1838$ & $3 \cdot 14135$ & $1 \cdot 138$ & 0.38 \\
\hline $0 \cdot 1806$ & 2.798 & 0.1684 & 0.044 & 0.2469 & $3 \cdot 105$ & 0.2692 & $3 \cdot 1395$ & $1 \cdot 614$ & 0.49 \\
\hline 0.2695 & $2 \cdot 644$ & 0.2430 & 0.09 & 0.3636 & 3.054 & 0.3922 & $3 \cdot 132$ & & \\
\hline $0 \cdot 4021$ & $2 \cdot 40$ & 0.3380 & 0.32 & 0.5394 & $2 \cdot 895$ & 0.5714 & 3.090 & & \\
\hline 0.5973 & $2 \cdot 10$ & 0.4210 & 1.73 & 0.8039 & $2 \cdot 78$ & 0.8358 & 2.976 & & \\
\hline 0.903 & 1.75 & 0.572 & $2 \cdot 32$ & $1 \cdot 139$ & $2 \cdot 5$ & $1 \cdot 1236$ & 2.75 & & \\
\hline 1.086 & 1.69 & 0.749 & $2 \cdot 49$ & & & & & & \\
\hline & & $1 \cdot 170$ & 2.25 & & & & & & \\
\hline
\end{tabular}

two s and one $z$ function on the fluorines and nine more $z$ functions at the midpoint to the HF basis. For the $\Pi_{u}$ calculations, one $x$-type Gaussian on each fluorine and eight more at the midpoint were added to the HF basis; one additional $x$ function on the fluorines and twelve more $x z$-type Gaussians at the midpoint were used in the $\Pi_{\mathrm{g}}$ diagonalization. The $\Delta_{\mathrm{g}}$ basis consisted of the HF basis plus six $x y$ Gaussians on each fluorine plus eight more at the midpoint.

Table 2 lists the $\mathrm{s}, \mathrm{p} \sigma, \mathrm{p} \pi, \mathrm{d} \pi$ and $\mathrm{d} \delta$ phaseshifts and corresponding eigenvalues. The number of digits given in the phaseshifts represents the number of significant digits that could be deduced from a region over which the phaseshift had stabilized. These figures merely reflect the stability of the extraction procedure and do not indicate the possible convergence of the phaseshift with respect to basis functions.

Since the eigenvalues corresponding to different symmetries cannot be made to coincide by a simple diagonalization of the Hamiltonian, the computed phaseshifts must be interpolated before cross sections can be calculated. These interpolated values were used to compute a total elastic cross section which satisfies the equation

$$
\sigma_{\mathrm{tot}}=\frac{4 \pi}{k^{2}} \sum_{l, m} \sin ^{2} \delta_{l m} .
$$

The total cross section is depicted in figure 1.

The cross section is seen to be dominated by a $\Sigma_{\mathrm{u}}^{+}-\mathrm{p} \sigma$ resonance at about $2 \cdot 2 \mathrm{eV}$ and rises from a background value of approximately $40 a_{0}^{2}$ to almost $120 a_{0}^{2}$ over an energy range of about $0.8 \mathrm{eV}$. Unfortunately, there were only five computed eigenvalues in the interesting region over which the po phaseshift rises through $2.4 \mathrm{rad}$, and thus the resonant cross section will be somewhat sensitive to the way in which the phaseshifts are interpolated.

\section{Discussion}

We have applied a simple, but approximate, method to calculate low-energy $\mathrm{e}^{-}-\mathrm{F}_{2}$ elastic cross sections. The calculations were carried out under the assumption of low $l$-spoiling and correlation and polarization effects were also ignored. Since the method is based solely on the diagonalization of the molecular Hamiltonian over 


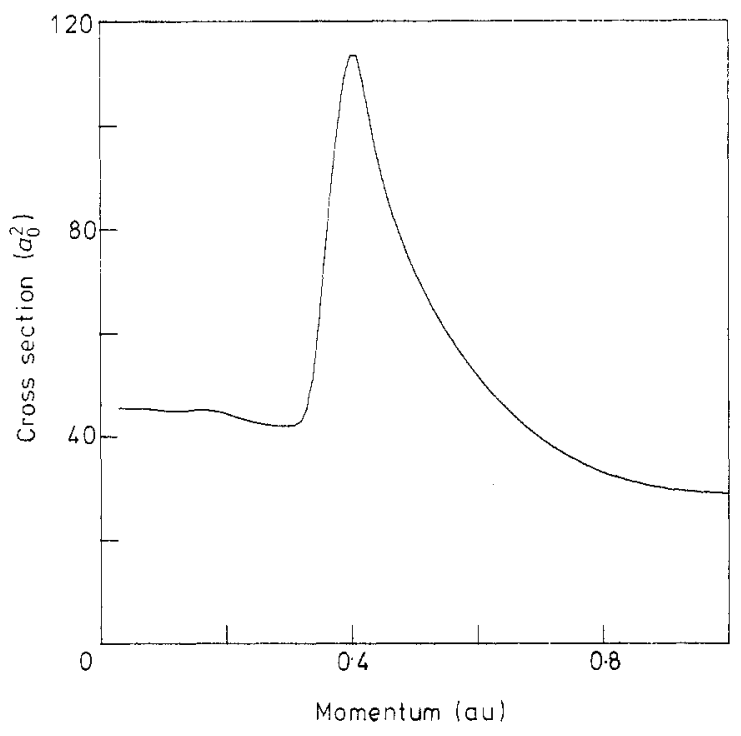

Figure 1. $\mathrm{e}^{-}-\mathbf{F}_{2}$ total elastic cross sections obtained from interpolated phaseshifts.

a discrete set of Gaussian basis functions and asymptotic boundary conditions are not explicity imposed, the entire procedure was implemented with standard boundstate molecular programs. Total cross sections were found by interpolation of phaseshifts obtained at discrete energy eigenvalues. The dominant feature we find in the elastic cross section is a $2 \cdot 2 \mathrm{eV} \Sigma_{\mathrm{u}}^{+}$shape resonance coming from the p $\sigma$ partial wave.

However, it is entirely possible that this resonance is an artifact of the staticexchange approximation and would disappear if correlation effects were included. It is interesting to note that a comparison of the Hartree-Fock potential energy curves for the ground states of $F_{2}$ (Das and Wahl 1966) and $F_{2}^{-}$(Gilbert and Wahl 1971) suggests that $F_{2}^{-}$is stable with respect to $F_{2}$ and a free electron at the equilibrium internuclear distance of $F_{2}$. If that were the case, the shape resonance found in the present static-exchange calculation would be of doubtful validity. However, if a multiconfiguration, self-consistent field (MCSCF) potential energy curve for $F_{2}$ (Das and Wahl 1972) is used in the above comparison, $\mathrm{F}_{2}^{-}$is predicted to be unstable at the equilibrium internuclear distance of $F_{2}$. Experimental evidence for the resonance may be inferred from studies on the dissociative attachment of low-energy electrons to $\mathrm{F}_{2}$; this work is summarized in a review by Caledonia (1975). The measured rate constants for $\mathrm{DA}$ to $\mathrm{F}_{2}$ are quite large, indicating that the reaction may well proceed through resonance excitation to an unbound $F_{2}^{-}$ion. In any event, the possibility of a stable negative ion suggests that the static-exchange approximation may be less valid for electron- $\mathrm{F}_{2}$ scattering than for the previously studied cases of $\mathrm{e}^{-}-\mathrm{H}_{2}$ and $\mathrm{e}^{-}-\mathrm{N}_{2}$ (McCurdy et al 1976). Since there are no other calculations or experiments with which to compare these results directly, confirmation of our findings must await further study. One important question that must be answered is whether, in view of the poor $\mathrm{HF}$ description of $F_{2}$, the use of a correlated $F_{2}$ ground state in the static-exchange approximation would significantly modify these results. Work along these lines is presently under study. 


\section{References}

Caledonia G E 1975 Chem. Rev. 75 333-51

Calgero F 1967 The Variable Phase Approach to Potential Scattering (New York: Academic Press)

Das G and Wahl A C 1966 J. Chem. Phys. 44 87-96

1972 J. Chem. Phys. 56 3532-40

Dunning T H $1970 \mathrm{~J}$. Chem. Phys. 53 2823-33

1971 J. Chem. Phys. 55 3958-66

Fano U 1970 Comments on Atomic and Molecular Physics 1 140-4

Gilbert T L and Wahl A C $1971 \mathrm{~J}$. Chem. Phys. 55 5247-61

Hazi A U 1973 Chem. Phys. Lett. 20 251-6

Hazi A U and Fels M F 1971 Chem. Phys. Lett. 18 582-6

Hazi A U and Taylor H S 1970 Phys. Rev. A 1 1109-20

McCurdy C W, Rescigno T N and McKoy V 1976 J. Phys. B: Atom. Molec. Phys. 9 691-8

Wahl A C 1964 J. Chem. Phys. 41 2600-11 